Check for updates

Cite this: J. Mater. Chem. C, 2020, 8, 15788

Received 3rd July 2020,

Accepted 1st October 2020

DOI: $10.1039 / \mathrm{dOtc03144e}$

rsc.li/materials-c

\section{A graphene-based electro-thermochromic textile display $\dagger$}

\author{
Xiaoqian Ji, ${ }^{\text {ab }}$ Wenwen Liu, ${ }^{a}$ Yunjie Yin, ${ }^{a}$ Chaoxia Wang (D) *a and Felice Torrisi (D) *bc
}

Electronic textiles (e textiles) are rapidly emerging as key enablers for wearable electronics. Graphene and 2D materials have played a major role in enabling truly wearable e-textiles. Here we demonstrate a textile-based display using the Joule's heating of a screen-printed, few-layer graphene ink to drive the colour switching of thermochromic polyurethane on a cotton fabric. The average temperature of the few-layer graphene ink on the fabric was voltage-controlled reaching about $43{ }^{\circ} \mathrm{C}$ in $45 \mathrm{~s}$ at a bias of $12 \mathrm{~V}$ and a recovery of $<20 \mathrm{~s}$ with negligible degradation after several heating/cooling cycles. This is used to demonstrate several electrothermochromic textile displays, thus representing a breakthrough in e-textiles technology.

\section{Introduction}

Wearable electronics is an emerging field that offers considerable potential in healthcare and well-being, Internet of Things (IoT) and biosensing applications. ${ }^{1-3}$ Electronic textiles (E-textiles) are a very promising technology that aims to revolutionize wearable electronics offering breathability, conformability and comfort to wear. ${ }^{4}$ Despite all-textile individual electronic components ${ }^{5}$ and integrated circuits have been demonstrated, ${ }^{6}$ only few reports have attempted to achieve textile-based displays. ${ }^{7}$ Thermochromic textile devices have already been demonstrated using metal oxide (such as $\mathrm{CoO}$ and $\mathrm{Pb}_{3} \mathrm{O}_{4}$ ) and leuco dye or polymer (such as polydyne) through various methods of textile integration such as coating, ${ }^{8}$ spinning, ${ }^{9}$ and dyeing. ${ }^{10}$

Thermochromic dyes are composed of thermochromic molecules (TMs). These molecules have the property to change in colour when exposed to an increase in the surrounding temperature (above a certain critical temperature, $T_{\mathrm{c}}$ ). The change in colour is attributed to the variation of the structural conformation of the molecule, induced by thermal energy. ${ }^{10}$ Thermochromic dyes have attracted considerable attention in medicine ${ }^{11}$ wearable devices ${ }^{12}$ and security materials because of their application as a storage element in memories,${ }^{13}$ temperature switches, ${ }^{14}$ colour changing paints ${ }^{15}$ and anti-counterfeiting devices. ${ }^{15,16}$ The leuco dye is one of the most common thermochromic dyes comprising a colour

\footnotetext{
${ }^{a}$ Key Laboratory of Eco-Textile, Ministry of Education, College of Textile Science and Engineering, Jiangnan University, Wuxi, 214122, China.

E-mail: wangchaoxia@sohu.com

${ }^{b}$ Cambridge Graphene Centre, Department of Engineering, University of Cambridge, 9 JJ Thomson Avenue, Cambridge CB3 OFA, UK. E-mail: ft242@cam.ac.uk,

f.torrisi@imperial.ac.uk

${ }^{c}$ Molecular Sciences Research Hub, Department of Chemistry, Imperial College London, 80 Wood Lane, London W12 OBZ, UK

$\dagger$ Electronic supplementary information (ESI) available. See DOI: 10.1039/d0tc03144e
}

former (i.e., conjugated molecule, which is responsible for the colour combination) and a developer (typically weak acids such as bisphenol A, gallates, hydroxybenzoates and hydroxycoumarin derivatives) in a non-volatile organic solvent (such as methyl stearate). ${ }^{17-19}$ The weight ratios of the colour former, the colour developer and the solvent is important for the engineering the thermochromic dye. If the solvent is too much, the thermochromic dye will be too light or even colourless below $T_{\mathrm{c}}$. However, if the solvent is too less, the thermochromic dye will change colour when above $T_{\mathrm{c}}$. Nevertheless, the presence of bisphenol A in leuco dyes generates major environmental concerns and solvent leakage upon heating ${ }^{20}$ (when the leuco dye reaches the thermochromic temperature, the solvent is changed to a liquid from a solid ${ }^{18}$ ), thus making them unsuitable for textile applications. ${ }^{21}$ Stimuliresponsive functional materials have been investigated as a suitable alternative. Ref. 22 combined red-green-blue (RGB) thermochromic materials (such as DEBN, cyan-6, yellow-1, and blue-220) and luminescent materials (such as Eu(III)(TTA) ${ }_{3}$ phen and $\mathrm{Alq}_{3}$ ) to obtain thermo-responsive dual-mode (defined as red-green-blue colouration and fluorescence) representations in RGB colours with large contrast (from RGB to white). A polyurethane-based fluorescent dye was synthesized by attaching a fluorescent dye (such as 4-amino- $N$-cyclohexyl-1,8naphthalimide) to polyurethane chains by capping reaction (through a reaction of $-\mathrm{NH}_{2}$ and $-\mathrm{NCO}$ ), resulting in an increase in fluorescence intensity above $T_{\mathrm{c}}{ }^{23}$ Thermochromic polyurethane is primarily prepared by mixing pure polyurethane with thermochromic dyes. A waterborne UV-curable polyurethane acrylate/ $\mathrm{SiO}_{2}$ nanocomposites incorporating nanosilica were prepared using a new form of sol-gel method and mixed with inorganic thermochromic dyes to make a polyurethane-based thermochromic ink. ${ }^{8}$ Recently, ref. 24 demonstrated a thermochromic textile based on the UV-induction polyurethane coating mixed with a rhodamine thermochromic dye. The textile 
showed thermochromic behavior at $\sim 50{ }^{\circ} \mathrm{C}$ under magnetic heating with UV induction.

Moreover, electrically driven thermochromic switching was proposed by ref. 25 The device was obtained by weaving metallic yarns within the fabrics and then painting thermochromic inks on the top of them. Ref. 26 deposited a thermochromic ink on one side of a textile substrate and used metal wires as heating elements on the other side, thus achieving a temperature, $T \sim 50{ }^{\circ} \mathrm{C}$ at a voltage bias $\left(V_{\text {bias }}\right)$ of $7 \mathrm{~V}$. Ref. 27 also prepared an electro-thermochromic paper with a commercial leuco dye as the thermochromic ink, which achieved switching at $T \sim 45{ }^{\circ} \mathrm{C}$ at $V_{\text {bias }} \sim 4 \mathrm{~V}$.

However, this strategy has its own limitations because the colour change can only happen in the region close to the conductive yarn when a current passes through it. Recently, the combination of electric heaters from conductive coatings and colour-changing dyes from TMs has been exploited to demonstrate an electrothermochromic textile. This process allows the coating or printing of specific patterns, thus enabling electro-thermochromic image and text. For example, ref. 28 described the production of conductive non-woven mats of poly-3,4-ethylenedioxythiophene (PEDOT) using electrospinning. In this case, when an electric current passes through the PEDOT film producing sufficient heat $\left(T \sim 100{ }^{\circ} \mathrm{C}\right.$ at $\left.V_{\text {bias }} \sim 10 \mathrm{~V}\right)$, the coated thermochromic ink undergoes a colour change. Recently, an electromagnetic interference shielding fabric and stable Joule heating response based on CNTs network-embedded poly(vinyl alcohol) (PVA) composite film were reported..$^{29-31}$ More recently, ref. 32 prepared a coaxial multilayer monofilament whose conductive core layer could trigger the colour change of the external thermochromic layer on resistive heating. However, the conductivity of the monofilament is low $\left(1 \times 10^{-2} \mathrm{~S} \mathrm{~cm}^{-1}\right)$, requiring a high voltage to induce a thermochromic colour change and becoming unsuitable for wearable applications. Graphene and graphene nano-platelets (GNPs) have been the focus of intense research as suitable materials for electric heating, ${ }^{33}$ as well as biocompatible ${ }^{34,35}$ and wearable electronics. ${ }^{6,36,37}$

Based on previous works on UV-induced thermochromic textiles ${ }^{24}$ using rhodamine B ethylenediamine derivative molecules, we demonstrated a screen-printed electro-thermochromic textile (ET) on the cotton fabric comprising a UV-induced thermochromic polyurethane layer based on the rhodamine $\mathrm{B}$ ethylenediamine derivative molecules and GNP electrodes.
The electro-thermochromic switching of the fabric from magenta to white was controlled via Joule heating from an applied voltage, reaching $T \sim 43{ }^{\circ} \mathrm{C}$ at $V_{\text {bias }} \sim 12 \mathrm{~V}$ within $45 \mathrm{~s}$ and a recovery of $<20$ s. We then used this technology to demonstrate an electrothermochromic textile display (ETD).

\section{Results and discussion}

The voltage-controlled heating of the screen printable GNP ink is important to enable the electro-thermochromic devices on the textile. Here, we first fabricate the electrically and thermally conducting fabric by screen printing the GNP ink on a cotton fabric and then investigate the surface morphology, the electrical properties of the printed GNP ink, and the performance as a textile heater.

\section{Graphene nano-platelets ink}

The GNP ink was prepared via the microfluidic exfoliation of graphite (see methods). Scanning electron microscopy (SEM) was used to investigate the surface topography and the lateral size of graphene flakes. Fig. 1(a) and (b) show SEM micrographs with the typical surface topography of the GNP ink. Statistics of the lateral size (defined as the longest dimension) of the GNP flakes (Fig. 3(c)) reveal that this peak is primarily observed at 1-1.5 $\mu \mathrm{m}$.

\section{Graphene nano-platelets fabric (GNP fabric)}

The white-coloured control cotton fabric is shown in Fig. 2(a). After the polyurethane treatment, the cotton fabric is dried in the oven at $60{ }^{\circ} \mathrm{C}$ for $10 \mathrm{~min}$ to remove any residual water, resulting in a layer film on the surface of the cotton fabric as Fig. 2(b). The GNP fabric (shown in Fig. 2(c)) is prepared by screen printing the GNP ink on a polyurethane (PU-)treated cotton fabric, thus forming a GNP ink layer ( $7.6 \mu \mathrm{m}$ thick) on the surface. Fig. 2(d)-(l) shows the optical SEM images of the control cotton fabric (Fig. 2(d), (g) and (j)), the PU-treated cotton fabric (Fig. 2(e), (h) and (k)) and the GNP fabric (Fig. 2(f), (i) and (l)) at different magnifications. The SEM images (Fig. 2(d), (g) and (j)) show a typically fibrous structure with the fibres twisting with each other, thus producing a quite rough fabric surface. Comparing Fig. 2(e) and (d), the surface of the cotton fabric is evenly covered with a film and the texture of
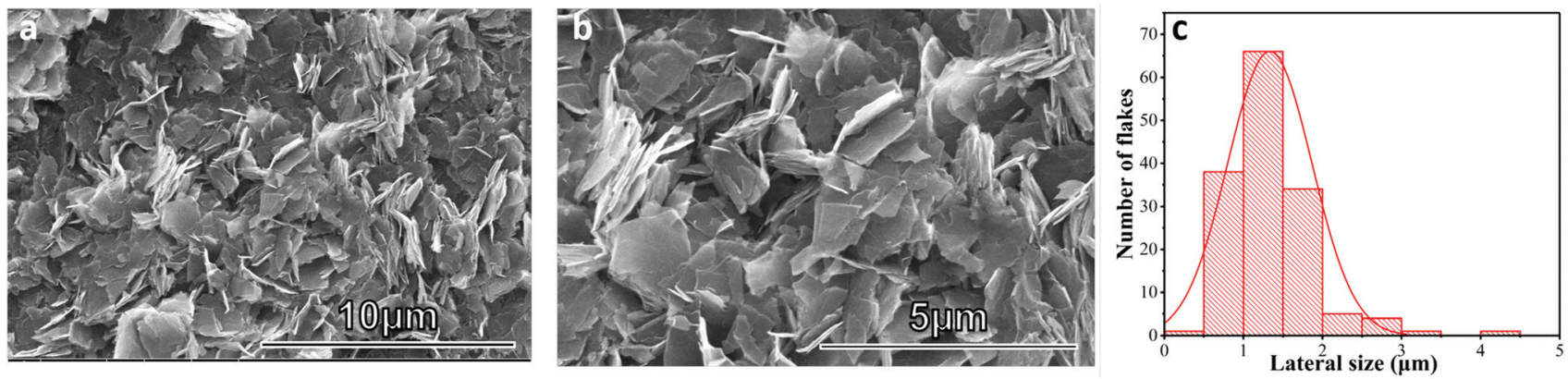

Fig. 1 (a) and (b) SEM images of GNP flakes; (c) Histogram of the lateral flake size for the GNP ink after 70 cycles of microfluidic exfoliation. 

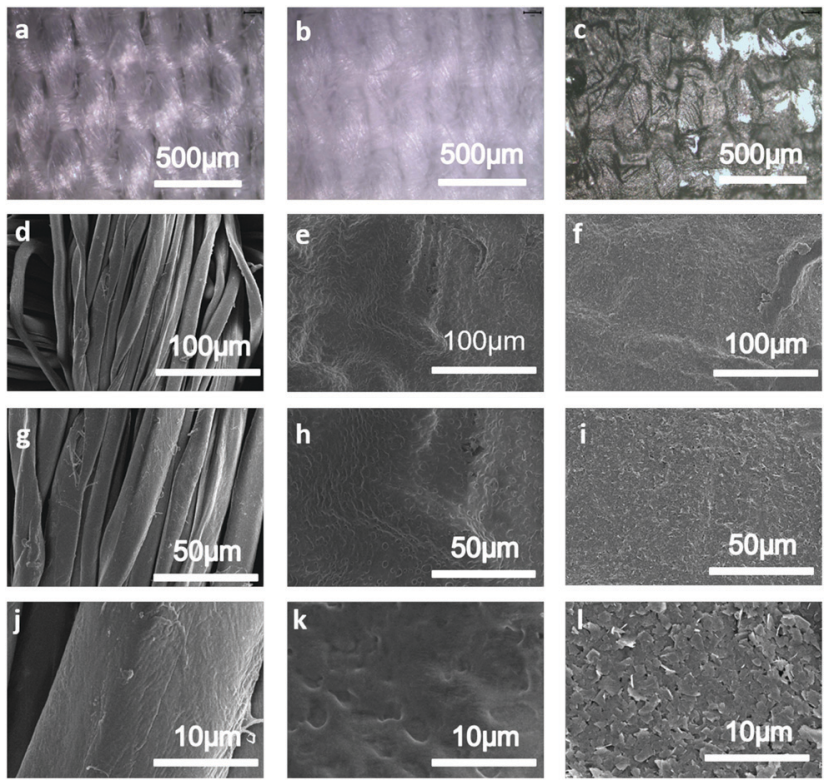

Fig. 2 Optical microscopy images of: (a) control cotton fabric, (b) PU-treated cotton fabric, (c) GNP fabric, SEM images of (d), (g), (j) control cotton fabric, (e), (h), (k) PU-treated cotton fabric, (f), (i), (l) GNP fabric.

the fabric is faintly seen. The surface of the pre-treated cotton fibre is also covered with a layer of polyurethane film. The SEM image in Fig. 2(f) shows how the GNP ink uniformly conforms to the pre-treated cotton fabric. Compared with the control cotton fabric, the carbon content of the GNP fabric was increased (shown as Fig. S1, ESI $\dagger$ ).

(a)
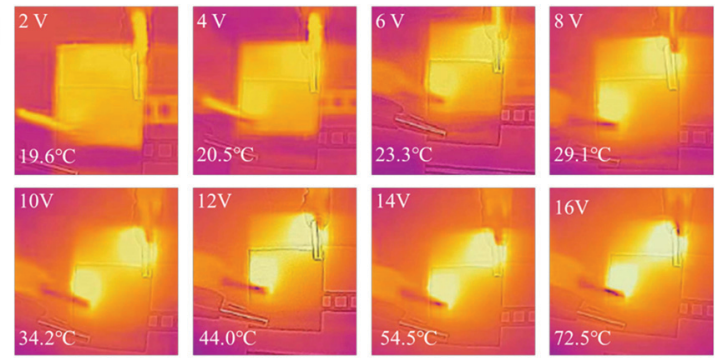

(b)

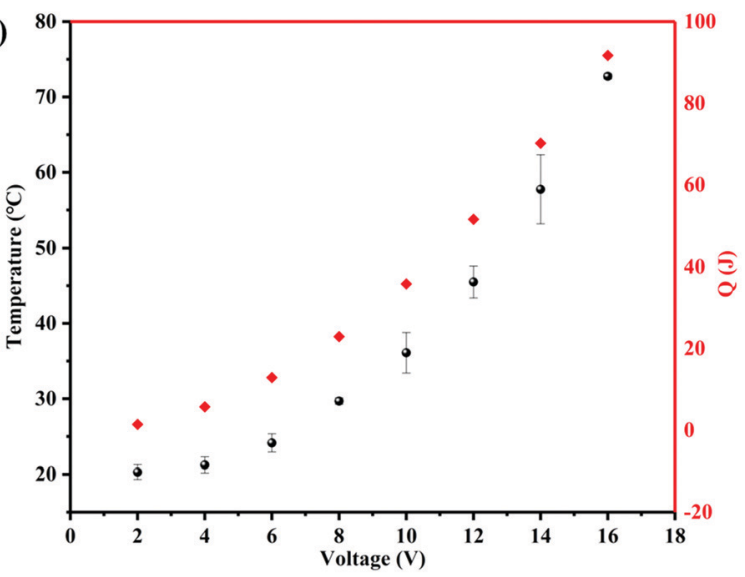

Fig. 3 (a) Infrared thermal images of the conductive fabric at various $V_{\mathrm{GNP}}$; (b) plot of the temperature versus $V_{\mathrm{GNP}}$.
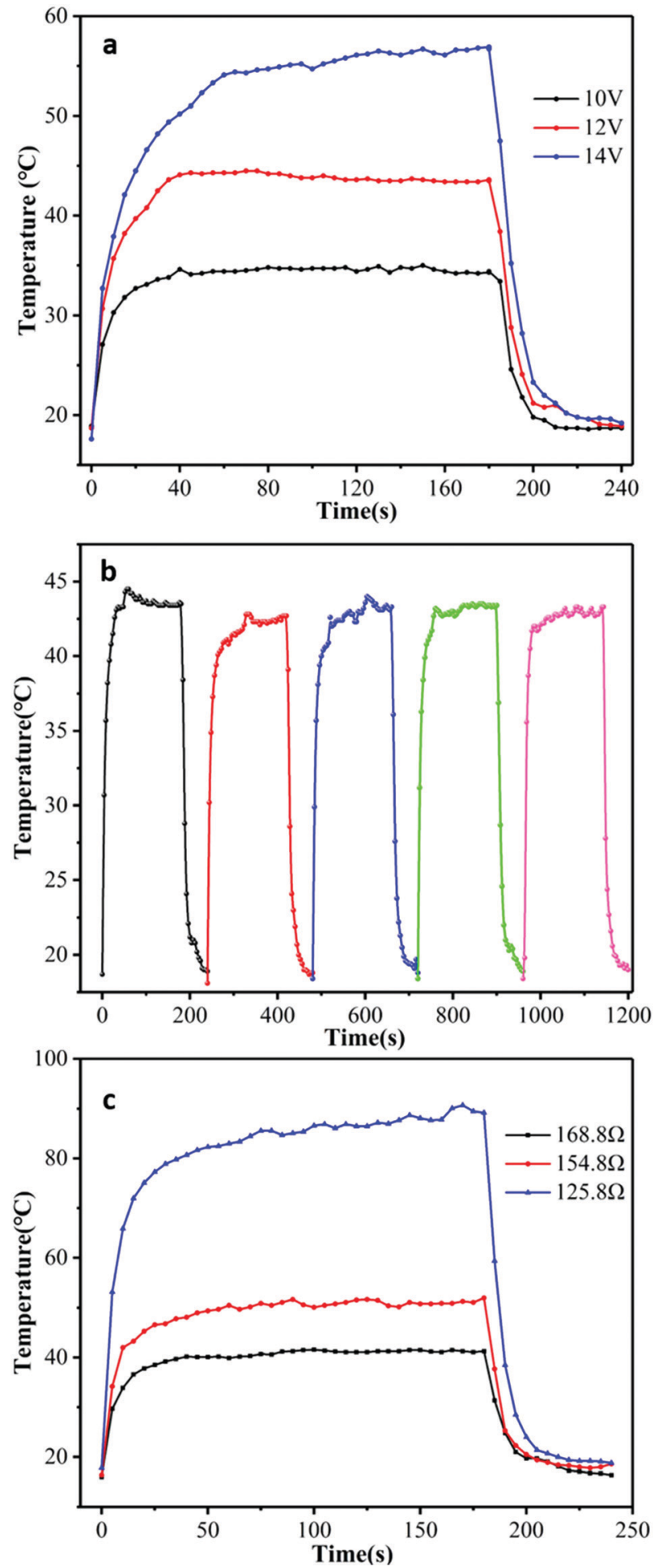

Fig. 4 (a) Temperature profiles of the GNP fabric at different $V_{\mathrm{GNP}}$; (b) temperature response of the GNP fabric over 5 cycles at $V_{G N P}=12 \mathrm{~V}$, each cycles is 4 min long; (c) temperature profiles of the GNP fabric operated at different biases.

\section{Electrical and thermal characterization of the GNP fabric}

The electrical and heating properties of the GNP fabric are characterized on a $2 \times 2 \mathrm{~cm}$ sample, thus revealing a sheet resistance of $R_{\mathrm{S}} \sim 169 \Omega$ and conductivity of $\sigma \sim 778.6 \mathrm{~S} \mathrm{~m}^{-1}$, 
which is consistent with the conductivity values reported by previous works. ${ }^{35,38}$ The heating properties of the GNP fabric were investigated by monitoring the evolution of the temperature by an infrared camera (see methods) as a function of the applied voltage $\left(V_{\mathrm{GNP}}\right)$ across the GNP fabric (between $2 \mathrm{~V}$ and $16 \mathrm{~V}$; Fig. 3). The thermal images show a clear heating of the GNP fabric as a function of $V_{\mathrm{GNP}}$, increasing from $T \sim 19.6{ }^{\circ} \mathrm{C}$ to $\sim 72.5{ }^{\circ} \mathrm{C}$.

The heating of our GNP fabric can be described by Joule's law, which defines the heat $(Q)$ dissipated from a resistive material when voltage (or current) is applied as follows: ${ }^{39,40}$

$$
Q=V^{2} / R t
$$

where $V$ is the applied voltage, $R=335 \Omega$ is the resistance of the conductive fabric (see methods) and $t$ is the operating time. The measured temperature change and calculated $Q$ as a function of voltage is shown in Fig. 3(b). Temperature profiles of the conductive fabric under different biases are measured as shown in Fig. S2 (ESI $\dagger$ ). At $V_{\mathrm{GNP}}=6 \mathrm{~V}$, only $\sim 19 \%$ increase of temperature was detected on the GNP-printed pattern. A change of regime takes place at a higher voltage and the temperature began to significantly rise from $T=\sim 23.3{ }^{\circ} \mathrm{C}$ at $V_{\mathrm{GNP}}=6 \mathrm{~V}$ to $T=\sim 72.5{ }^{\circ} \mathrm{C}$ at $V_{\mathrm{GNP}}=16 \mathrm{~V}$. This large temperature modulation of the GNP fabric was ascribed to the remarkable electrical conductivity of the GNP ink and the heat dissipation of cotton fibres.

Fig. 4(a) shows the temperature profiles of the GNP fabric, as a function of $t$ for $V_{\mathrm{GNP}}=10 \mathrm{~V}, 12 \mathrm{~V}$ and $14 \mathrm{~V}$. All curves show a similar trend with a time response $(\tau$, defined as the time required to reach the steady-state temperature, which was above $90 \%$ of the highest temperature from room temperature) of $\tau=45 \mathrm{~s}, \tau=45 \mathrm{~s}$ and $\tau=60 \mathrm{~s}$ for $V_{\mathrm{GNP}}=10 \mathrm{~V}, 12 \mathrm{~V}$ and $14 \mathrm{~V}$, respectively. In this case, when $V_{\mathrm{GNP}}=10 \mathrm{~V}$, only $T \sim 15{ }^{\circ} \mathrm{C}$ increase was observed, reaching $T \sim 35{ }^{\circ} \mathrm{C}$ after $\tau=40 \mathrm{~s}$. At $V_{\mathrm{GNP}}=14 \mathrm{~V}$, the GNP fabric reached $T \sim 60{ }^{\circ} \mathrm{C}$ after $\tau=60 \mathrm{~s}$.
The steady-state temperatures were reached for all samples in $<60 \mathrm{~s}$, thus demonstrating a faster response ${ }^{41,42}$ than graphenebased electro-thermal films, which reaches $T \sim 42{ }^{\circ} \mathrm{C}$ when $60 \mathrm{~V}$ is applied for 2 min. ${ }^{43}$ In all our GNP fabrics, the temperature remained stable on constant voltage applied and then returned to room temperature $\left(T \sim 20{ }^{\circ} \mathrm{C}\right)$ after about $60 \mathrm{~s}$ when power was turned off. This cooling rate can be explained by the nature of the cotton fabric that allows the heat to be very effectively dissipated by the material (the thermal conductivity of the cotton fabric is $0.026-0.065 \mathrm{~W} \mathrm{mK}^{-144}$ ).

To investigate the stability of the GNP fabric, repeated heating and cooling cycles were carried out (as show in Fig. 4(b) and Fig. S3, ESI $\dagger$ ). The temperature change of the GNP fabric was recorded over a period of $4 \mathrm{~min}$ (heating $3 \mathrm{~min}$ at $V_{\mathrm{GNP}}=12 \mathrm{~V}$ and cooling $1 \mathrm{~min}$ at $V_{\mathrm{GNP}}=0 \mathrm{~V}$ ) for five cycles. As shown in Fig. 4(b), the heating/cooling curve remained almost unchanged after several cycles, thus reaching constantly $T \sim 42{ }^{\circ} \mathrm{C}$ at $12 \mathrm{~V}$ for $40 \mathrm{~s}$ after several heating and cooling cycles, thus indicating a good stability and repeatability. During each heating process, the temperature increased from room temperature $\left(T \sim 20{ }^{\circ} \mathrm{C}\right)$ to a steady value of $T \sim 43{ }^{\circ} \mathrm{C}$ in the initial $1 \mathrm{~min}$, thus revealing a high heating rate of the GNP fabric. Moreover, in this case, the temperature of the GNP fabric gradually dropped to room temperature after $60 \mathrm{~s}$ because the voltage was set to $V_{\mathrm{GNP}}=0 \mathrm{~V}$ thanks to of the thermal conductivity of the cotton fabric. The time-dependent temperature profiles of the GNP fabrics with different $R_{\mathrm{S}}$ values are shown in Fig. 4(c). The temperature change of the GNP fabric was recorded with a period of $4 \mathrm{~min}$ (heating $3 \mathrm{~min}$ at $V_{\mathrm{GNP}}=12 \mathrm{~V}$ and cooling $1 \mathrm{~min}$ at $V_{\mathrm{GNP}}=0 \mathrm{~V}$ ). The heat dissipated, $Q$, is influenced by the resistance of the GNP fabric. At the same voltage, the temperature of the GNP fabric is inversely proportional to $R_{\mathrm{S}}$, thus confirming the heating by the Joule effect of the GNP fabric. For $R_{\mathrm{S}} \sim 126 \Omega$, the temperature of the GNP fabric reaches $T \sim 90{ }^{\circ} \mathrm{C}$, while it only reaches about $40{ }^{\circ} \mathrm{C}$ for $R_{\mathrm{S}} \sim 169 \Omega$. The conductivity of our GNP fabric is higher than that

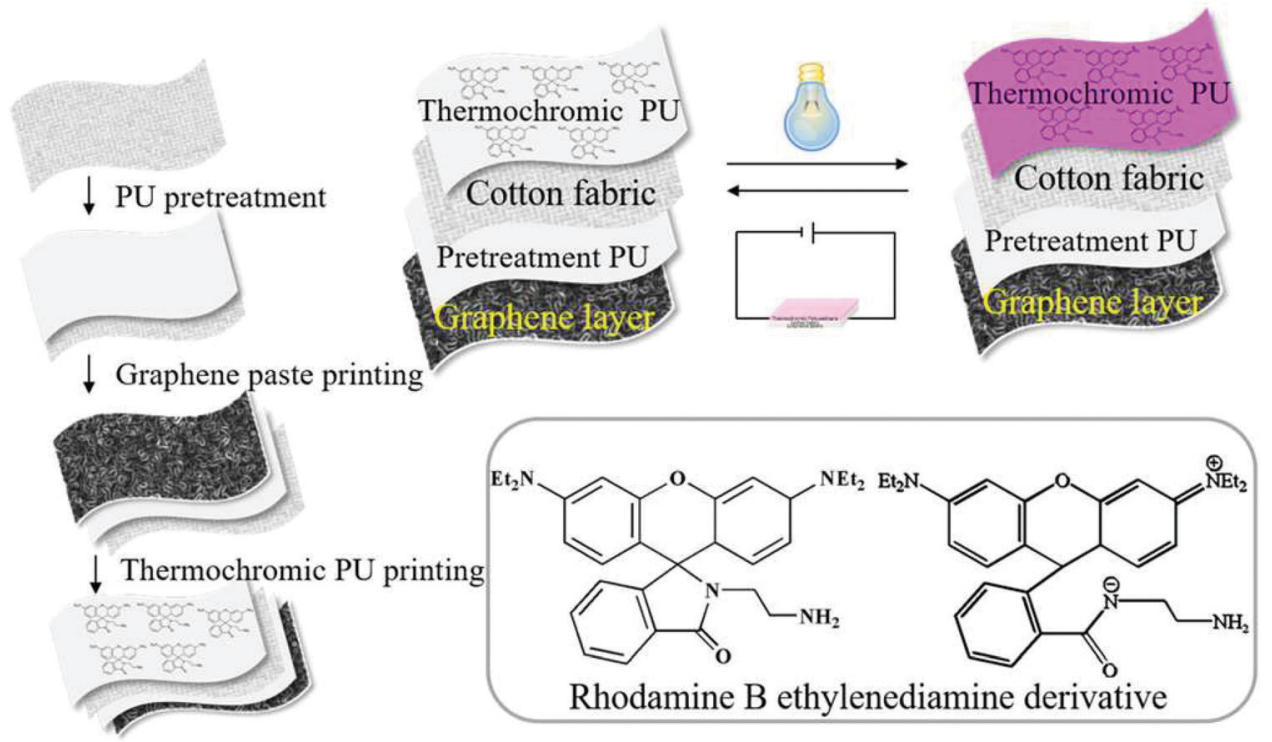

Fig. 5 The schematic diagram of the procedure used to fabricate the ET. In the frame the molecular structure of the two rhodamine B derivatives. 
reported for graphene anodes and cathodes (e.g., in microbial fuel cells). ${ }^{35}$

\section{Electro-thermochromic cotton fabric performance}

We developed a colour-changing smart fabric and a textile display by combining the colour changing property of the thermochromic polyurethane ink and the GNP fabric heater, as shown in Fig. 5. The rhodamine B ethylenediamine derivative thermochromic polyurethane ink was screen-printed on one side of the PU-treated cotton fabric, while the GNP ink was screen-printed on the other side (Fig. 5). The choice of depositing the two inks on the opposite sides aims to maximize the colour changing property of the thermochromic dye. The layer of polyurethane reduced the surface roughness ${ }^{38}$ of the fabric and prevented the absorption of the inks into the cotton fabric. The colour-changing property of the ET was investigated by monitoring colour parameters (i.e., $K / S$, where $a^{*}$ corresponds to the red and green colour combination and $b^{*}$ corresponds to yellow and blue colour combination) as a function of $V_{\mathrm{GNP}}$. The darker the red, the larger the $a^{*}$. Fig. 6(a) shows how the colour of the thermochromic coating gradually disappears as the applied voltage increases.

This is the result of the UV induction, which modifies the conjugate structure of the rhodamine $\mathrm{B}$ ethylenediamine derivative in the chemical composition of the polyurethane ink, thereby resulting in a change of the polyurethane optical properties. ${ }^{45}$ Our transparent thermochromic polyurethane starts reflecting in the region around magenta $\left(\lambda_{\max }=550 \mathrm{~nm}\right)$ after $\mathrm{UV}$ induction. It then returns colourless upon heating. The optical images of our ET at different voltages are shown in Fig. 6(a). Firstly, the thermochromic coating on the ET was activated by a treatment irradiation at $\lambda=254 \mathrm{~nm}$ for $3 \mathrm{~min}$. This allows the lactam ring structure of the rhodamine B ethylenediamine derivative moiety in the thermochromic polyurethane to gradually change to an open-loop state with a quinolone structure. ${ }^{24}$ Then, we applied an increasing voltage from $V_{\mathrm{GNP}}=2 \mathrm{~V}$ to $V_{\mathrm{GNP}}=16 \mathrm{~V}$. We can see the ET returning white as the applied voltage increased above $V_{\mathrm{GNP}}=10 \mathrm{~V}$. This is because the $Q$ generated by the GNP ink is sufficient to degrade the quinolone structure in the thermochromic ink, backconverting it into the colourless lactam ring when $V_{\mathrm{GNP}}>10-12 \mathrm{~V}$. A $V_{\mathrm{GNP}}=12 \mathrm{~V}$ corresponds to a higher temperature than $T_{\mathrm{c}}$, which consistently yields a transparent thermochromic coating. In the heating process, the GNP coating is the heat source, which transfers heat to the thermochromic polyurethane by radiation and conduction, as shown in the schematic of ET in Fig. 5.

\section{An electro-thermochromic textile display}

A series of ETDs are prepared by screen printing the GNP ink following the steps in Fig. 5 (Fig. 6(b)-(d)). The thermochromic polyurethane ink was screen-printed on the other side of the PU pre-treated cotton fabric with a series of predetermined patterns, "JNU" (Fig. 6(b)), a horseshoe (Fig. 6(c)) and a butterfly (Fig. 6(d)). The thermochromic pattern is then activated by UV induction at $\lambda=254 \mathrm{~nm}$, thus giving rise to the patterns in magenta. A voltage $V_{\mathrm{GNP}}>12 \mathrm{~V}$ turns the patterns colourless, as shown in Fig. 6(b)-(d). Moreover, also in this case, we attribute the colour change of the a
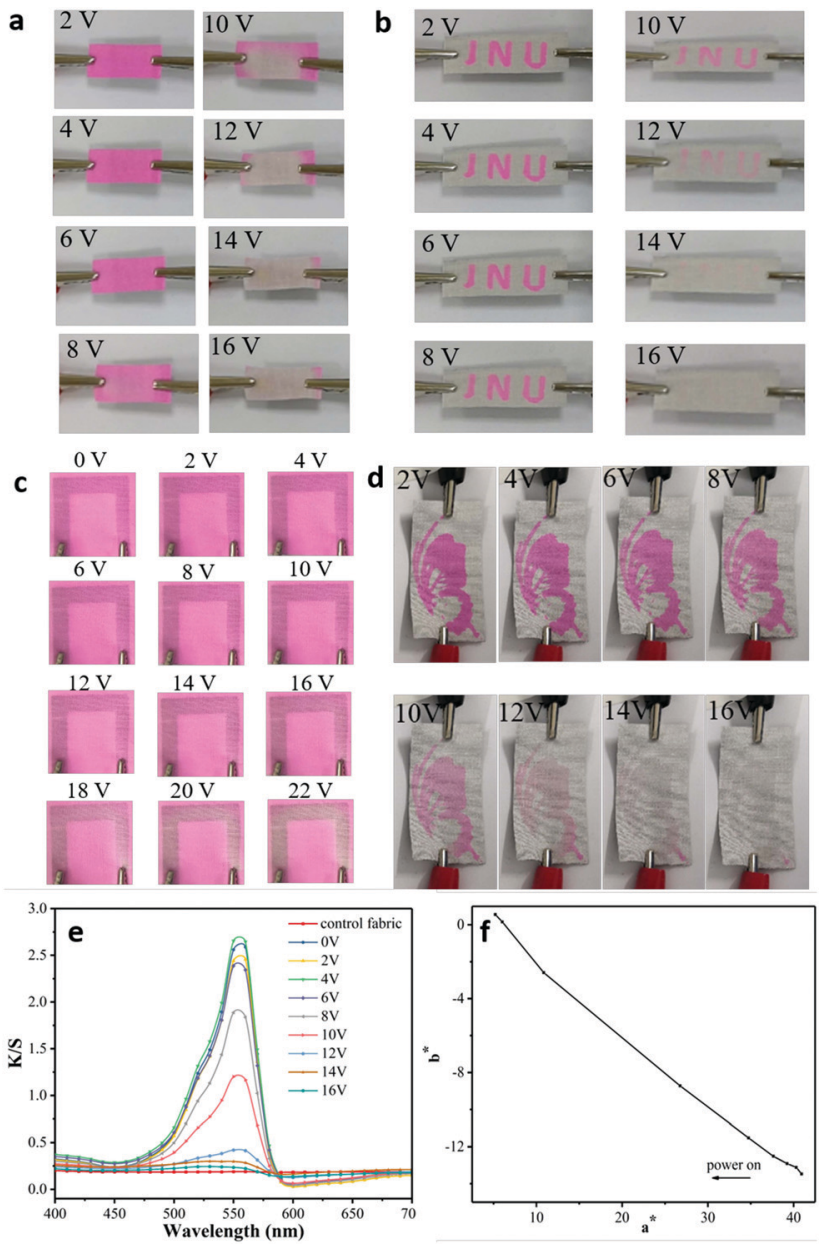

Fig. 6 (a) Photographs of ET $(1 \mathrm{~cm} \times 2 \mathrm{~cm})$ showing smart colourchanging effect as a function of $V_{\mathrm{GNP}}$; (b) photographs of ET $(1 \mathrm{~cm} \times$ $3 \mathrm{~cm}$ ) showing smart colour-changing effect as a function of $V_{\mathrm{GNP}}$; (c) photographs of ET with a horseshoe pattern, showing smart colourchanging effect as a function of $V_{\mathrm{GNP}}$; (d) photographs of ET with butterfly pattern, showing smart colour-changing effect as a function of $V_{\mathrm{GNP}}$; the (e) The $K / S$ curves of the ET as a function of $V_{\mathrm{GNP}}$; (f) the $a^{*}$ and $b^{*}$ of the ET as a function of $V_{G N P}$.

printed pattern to the Joule heat generated by the GNP ink printed at the back of the fabric. Fig. 6(e), (f) and Table S1 (ESI $\dagger$ ) show the colour parameters (colour difference, $\Delta E$, the $K / S$ value, $a^{*}, b^{*}$ ) of the ET as a function of $\lambda$ for a $V_{\mathrm{GNP}}$ range between 2 and $16 \mathrm{~V}$. The $K / S$ value shows peaks at $560 \mathrm{~nm}$, which are in agreement with the standard peaks for the rhodamine $\mathrm{B}$ ethylenediamine derivative. In particular, the reference peak at $\lambda=560 \mathrm{~nm}$ decreased as the voltage increased and $a^{*}$ decreased, which confirms the colour change of the ET and the patterned ETD from magenta to white.

\section{Conclusion}

We obtained electrochromic cotton fabrics and electrochromic textile-based displays using the thermal dissipation of a screenprinted, graphene-based ink to drive the colour switching of the thermochromic polyurethane ink coated on a fabric. The surface of a polyurethane fabric was covered by a graphene nanoplatelets 
ink uniformly. The graphene-based fabric-generated heat by the Joule effect upon voltage bias reached $T \sim 43{ }^{\circ} \mathrm{C}$ at $12 \mathrm{~V}$. When further coated by thermochromic polyurethane and activated by UV induction, the composite fabric switched from magenta to white upon an applied voltage of $10 \mathrm{~V}$, thus demonstrating suitable electro-thermochromic switching. The structure of the thermochromic polyurethane was changed to an open-loop state with a quinolone structure and returned to white when the applied voltage increased with the close-loop state of thermochromic polyurethane. This work paves the way for large-area, fast and stable thermochromic textile displays in future.

\section{Methods}

\section{Materials}

The UV-induced thermochromic polyurethane ink was homemade ${ }^{24}$ (more details in S2, ESI $\dagger$ ). Timrex KS25 graphite, sodium deoxycholate (SDC) and carboxymethyl cellulose sodium (CMC) were supplied by Aldrich Chemical Co Inc. The water-soluble thermal was purchased from Jining Baichuan Chemical Co., Ltd. The printing thickener was supplied by Transfar Co., Ltd.

\section{Formulation of the graphene-based ink}

The GNP ink was prepared by dispersing graphite flakes (Timrex KS25) in deionized water (DIW) with sodium deoxycholate (SDC, $10 \mathrm{mg} \mathrm{mL} \mathrm{mL}^{-1}$ ) followed by a microfluidic exfoliation (M110-P Microfluidizer; Microfluidics) ${ }^{6}$ for 70 cycles, thus obtaining a graphene-based dispersion. Sodium carboxymethyl cellulose (CMC; $7.5 \mathrm{~g} \mathrm{~L}^{-1}$ ) was blended with the graphene-based dispersion at $60{ }^{\circ} \mathrm{C}$ to obtain a GNP ink suitable for screen printing.

Fabrication of the electro-thermochromic cotton fabric and display The electro-thermochromic cotton fabric ET was prepared by screen printing on a polyurethane-coated cotton fabric, the graphene heater electrode on the back-side of the fabric, and the thermochromic UV-induced polyurethane on the front-side of the fabric by the following steps.

\section{Cotton fabric pre-treatment}

The cotton fabric was pre-treated with water-soluble thermal curing polyurethane (65\%), DIW (33\%) and thickener (2\%). Then, the cotton fabric was oven dried at $60{ }^{\circ} \mathrm{C}$ for $10 \mathrm{~min}$ and $100{ }^{\circ} \mathrm{C}$ for $5 \mathrm{~min}$ to prepare the pre-treated cotton fabric, denoted as PU pre-treated cotton fabric.

\section{Printing of GNP ink}

The GNP ink is deposited via screen-printing on the polyurethanetreated cotton fabric, followed by a drying step at $60{ }^{\circ} \mathrm{C}$ for $10 \mathrm{~min}$. The conductive fabric was denoted as the GNP fabric.

\section{Printing of thermochromic polyurethane}

Screen-printing was performed with the UV induction thermochromic polyurethane on the back of the conductive fabric. Then, the electro-thermochromic fabric was dried at $60{ }^{\circ} \mathrm{C}$ for $10 \mathrm{~min}$ and baked at $130{ }^{\circ} \mathrm{C}$ for $5 \mathrm{~min}$.

\section{Electron microscopy characterization}

The original graphene ink was dried to prepare the sample for scanning electron microscopy. The surface morphology of the conductive fabric was investigated by SEM. A statistical analysis of over 150 flakes of the original GNP ink was used to estimate the statistics of the lateral size (defined as the longest side of the flake).

\section{Sheet resistance measurements}

The sheet resistance of the conductive fabric was measured by a four-point probe system (KEITHLEY 2400 Source Meter Unit, US). The final resistance value of the fabric was measured with 10 different positions and averaged.

\section{Thickness measurements}

The thickness of the GNP flakes was measured with a fabric thickness test equipment (YG141, Changzhou Second Textile Machinery Factory, China).

\section{Thermal and electro-thermochromic measurements}

The average temperature of the fabric surface was monitored with an infrared camera (FLIR Ex). The infrared images of the conductive fabric were acquired at various $V_{\mathrm{GNP}}$ applied across the GNP, with an exposure time of $2 \mathrm{~min}$. The electrothermochromic behaviour of the GNP fabric was investigated at various $V_{\mathrm{GNP}}$ applied across the ET and ETDs.

\section{Colour parameters}

The colour parameters $\left(K / S\right.$ value, $\left.L^{*}, a^{*}, b^{*}, C^{\circ}, h^{\circ}\right)$ of the ETs and ETDs were acquired at various $V_{\mathrm{GNP}}$ using Xrite-8400 spectrophotometer under a D65 illumination using a $10^{\circ}$ standard observer.

\section{Conflicts of interest}

There are no conflicts to declare.

\section{Acknowledgements}

The authors acknowledge funding from the National First-Class Discipline Program of Light Industry Technology and Engineering (LITE2018-21), the 111 Project (B17021), the National Natural Science Foundation of China (No. 21975107), Fundamental Research Funds for the Central Universities (JUSRP51724B), the International Joint Research Laboratory for Advanced Functional Textile Materials, the China Scholarship Council (201706790076 and 201806790073), EPSRC grants EP/P02534X/2, EP/R511547/1, EP/T005106/1 and Trinity College, Cambridge. We wish to thank Dr Tian Carey and Mr Adrees Arbab for useful discussions.

\section{References}

1 J. Chen, Q. Peng, T. Thundat and H. Zeng, Chem. Mater., 2019, 31, 4553-4563.

2 Y. Guo, C. Dun, J. Xu, J. Mu, P. Li, L. Gu, C. Hou, C. A. Hewitt, Q. Zhang, Y. Li, D. L. Carroll and H. Wang, Small, 2017, 13, 1-9. 
3 J. A. Rogers, T. Someya and Y. Huang, Science, 2010, 327, 1603-1607.

4 R. Cao, X. Pu, X. Du, W. Yang, J. Wang, H. Guo, S. Zhao, Z. Yuan, C. Zhang, C. Li and Z. L. Wang, ACS Nano, 2018, 12, 5190-5196.

5 D. Na, J. Choi, J. Lee, J. W. Jeon and B. H. Kim, ACS Appl. Mater. Interfaces, 2019, 11, 27353-27357.

6 P. G. Karagiannidis, S. A. Hodge, L. Lombardi, F. Tomarchio, N. Decorde, S. Milana, I. Goykhman, Y. Su, S. V. Mesite, D. N. Johnstone, R. K. Leary, P. A. Midgley, N. M. Pugno, F. Torrisi and A. C. Ferrari, ACS Nano, 2017, 11, 2742-2755.

7 S. Yao, J. Yang, F. R. Poblete, X. Hu and Y. Zhu, ACS Appl. Mater. Interfaces, 2019, 11, 31028-31037.

8 C. Lv, L. Hu, Y. Yang, H. Li, C. Huang and X. Liu, RSC Adv., 2015, 5, 25730-25737.

9 Y. Lu, X. Xiao, Z. Cao, Y. Zhan, H. Cheng and G. Xu, Appl. Surf. Sci., 2017, 425, 233-240.

10 W. Zhang, X. Ji, C. Zeng, K. Chen, Y. Yin and C. Wang, J. Mater. Chem. C, 2017, 5, 8169-8178.

11 A. C. Siegel, S. T. Phillips, B. J. Wiley and G. M. Whitesides, Lab Chip, 2009, 9, 2775-2781.

12 Y. Li, Z. Zhang, X. Li, J. Zhang, H. Lou, X. Shi, X. Cheng and H. Peng, J. Mater. Chem. C, 2017, 5, 41-46.

13 I. J. Kim, M. Ramalingam and Y. A. Son, Dyes Pigm., 2018, 151, 64-74.

14 M. Basson and T. S. Pottebaum, Exp. Fluids, 2012, 53, 803-814.

15 S. Hirata, K. S. Lee and T. Watanabe, Adv. Funct. Mater., 2008, 18, 2869-2879.

16 L. S. Ribeiro, T. Pinto, A. Monteiro, O. S. G. P. Soares, C. Pereira, C. Freire and M. F. R. Pereira, J. Mater. Sci., 2013, 48, 5085-5092.

17 C. Yu, Y. Zhang, D. Cheng, X. Li, Y. Huang and J. A. Rogers, Small, 2014, 10, 1266-1271.

18 A. Raditoiu, V. Raditoiu, C. A. Nicolae, M. F. Raduly, V. Amariutei and L. E. Wagner, Dyes Pigm., 2016, 134, 69-76.

19 S. Ayazi-Yazdi, L. Karimi, M. Mirjalili and M. Karimnejad, J. Text. Inst., 2017, 108, 856-863.

20 X. Geng, W. Li, Y. Wang, J. Lu, J. Wang, N. Wang, J. Li and X. Zhang, Appl. Energy, 2018, 217, 281-294.

21 F. Azizian, A. J. Field and B. M. Heron, Dyes Pigm., 2013, 99, 431-439.

22 K. Ogasawara, K. Nakamura and N. Kobayashi, J. Mater. Chem. C, 2016, 4, 4805-4813.

$23 \mathrm{X} . \mathrm{Hu}, \mathrm{X}$. Zhang, J. Liu and J. Dai, Polym. Int., 2014, 63, 453-458.

24 X. Ji, W. Zhang, F. Ge, C. Wang, Y. Yin and K. Chen, Prog. Org. Coat., 2019, 131, 111-118.
25 A. Bhattacharyya and M. Joshi, Fibers Polym., 2011, 12, 734-740.

26 K. R. Karpagam, K. S. Saranya, J. Gopinathan and A. Bhattacharyya, J. Text. Inst., 2017, 108, 1122-1127.

27 C. Wei, L. Fan, W. Rao, Z. Bai, W. Xu, H. Bao and J. Xu, Cellulose, 2017, 24, 5187-5196.

28 A. Laforgue, J. Mater. Chem., 2010, 20, 8233-8235.

29 B. Zhou, Y. Li, G. Zheng, K. Dai, C. Liu, Y. Ma, J. Zhang, N. Wang, C. Shen and Z. Guo, J. Mater. Chem. C, 2018, 6, 8360-8371.

30 B. Zhou, M. Su, D. Yang, G. Han, Y. Feng, B. Wang, J. Ma, J. Ma, C. Liu and C. Shen, ACS Appl. Mater. Interfaces, 2020, 12, 40859-40869.

31 B. Zhou, X. Han, L. Li, Y. Feng, T. Fang, G. Zheng, B. Wang, K. Dai, C. Liu and C. Shen, Compos. Sci. Technol., 2019, 183, 107796.

32 A. Laforgue, S. Dubost, M. F. Champagne and L. Robitaille, ACS Appl. Mater. Interfaces, 2012, 4, 3136-3168.

33 H. Sun, D. Chen, C. Ye, X. Li, D. Dai, Q. Yuan, K. W. A. Chee, P. Zhao, N. Jiang and C. Te Lin, Appl. Surf. Sci., 2018, 435, 809-814.

34 F. Torrisi, D. Popa, S. Milana, Z. Jiang, T. Hasan, E. Lidorikis and A. C. Ferrari, Adv. Opt. Mater., 2016, 4, 1088-1097.

35 T. P. Call, T. Carey, P. Bombelli, D. J. Lea-Smith, P. Hooper, C. J. Howe and F. Torrisi, J. Mater. Chem. A, 2017, 5, 23872-23886.

36 J. Ren, C. Wang, X. Zhang, T. Carey, K. Chen, Y. Yin and F. Torrisi, Carbon N. Y., 2017, 111, 622-630.

37 S. Qiang, T. Carey, A. Arbab, W. Song, C. Wang and F. Torrisi, Nanoscale, 2019, 11, 9912-9919.

38 T. Carey, S. Cacovich, G. Divitini, J. Ren, A. Mansouri, J. M. Kim, C. Wang, C. Ducati, R. Sordan and F. Torrisi, Nat. Commun., 2017, 8, 1-11.

39 C. Celle, C. Mayousse, E. Moreau, H. Basti, A. Carella and J. P. Simonato, Nano Res., 2012, 5, 427-433.

40 W. Lan, Y. Chen, Z. Yang, W. Han, J. Zhou, Y. Zhang, J. Wang, G. Tang, Y. Wei, W. Dou, Q. Su and E. Xie, ACS Appl. Mater. Interfaces, 2017, 9, 6644-6651.

41 Y. Yu, W. Shen, F. Li, X. Fang, H. Duan, F. Xu, Y. Xiong, W. Xu and W. Song, RSC Adv., 2017, 7, 28670-28676.

42 G. Huang, L. Liu, R. Wang, J. Zhang, X. Sun and H. Peng, J. Mater. Chem. C, 2016, 4, 7589-7594.

43 D. Sui, Y. Huang, L. Huang, J. Liang, Y. Ma and Y. Chen, Small, 2011, 7, 3186-3192.

44 A. Abbas, Y. Zhao, J. Zhou, X. Wang and T. Lin, Fibers Polym., 2013, 14, 1641-1649.

45 Z. Wang, Z. Ma, Y. Wang, Z. Xu, Y. Luo, Y. Wei and X. Jia, Adv. Mater., 2015, 27, 6469-6474. 